\title{
QOE ENHANCED SOCIAL LIVE INTERACTIVE STREAMING
}

\author{
J.Ramya $^{1}$, D. Venkata Subramanian ${ }^{2}$, R.Nedunchellian ${ }^{3}$ \\ ${ }^{1}$ Dept of Computer Science \& Engineering, Saveetha School of Engineering, Saveetha University, Chennai, India. \\ ${ }^{2}$ Dept of Computer Science \& Engineering, Saveetha School of Engineering, Saveetha University, Chennai, India. \\ ${ }^{3}$ Dept of Computer Science \& Engineering, Saveetha School of Engineering, Saveetha University, Chennai, India.
}

\begin{abstract}
Globalized live streaming services have got subscribers all around the world. The rapid emergence of the mobile devices has made it a natural aspiration of such users to make social interaction with others who have got involved in the same application. The term Social TV implies the integrated support of the television and the computer technology in order to provide a group viewing experience to the users. The social interactions within the users who use such live streaming service needs to be spontaneous. The cloud computing technology has triggered enormous opportunities to facilitate the mobile live streaming of the multimedia contents with an extended support to interact with the users. The quality of service (QOS), storage and sharing are some of the issues that have to be addressed in order to provide a mobile social television in a cloud environment. The cloud technology effectively handles some of these issues by assigning proxies for the mobile users. These proxies (surrogates) for the users, operates on the base of transcoding mechanism. Also the PAAS and IAAS cloud services are keys in providing such an effective interaction based live streaming.
\end{abstract}

Keywords-Mobile Social TV, Live Streaming, Quality Of Service, Social Interactions, Cloud Computing. $* * *$

\section{INTRODUCTION}

Social TV started in the early 2000s with limited success as the creation of the shared connections was cumbersome with a remote control and the User Interface (UI) design made the interaction disruptive to the TV experience. But social networking has made Social TV suddenly feasible, since it already encourages constant connection between members of the network and the creation of likely minded groups. The shared content and activities often relate to TV content. At the same time, the smart phone market has been growing quickly. Mobile TV is the technology providing multimedia contents to the user by wireless communication. As our society is becoming increasingly mobile, there is an emerging need for content and services to become mobile as well. Within the scope of our research, we define Mobile TV as real-time transmission of traditional TV content to mobile handsets.

Mobile TV promises thrilling benefits to consumers and increased revenues for mobile telecommunications' operators, equipment suppliers and television providers. The mobiale TV device should produce an image quality which is not significantly inferior to the standard established by traditional $\mathrm{TV}$, even if the screen size is much smaller. The network coverage and the signal strength should be sufficiently good to give the viewer an uninterrupted service of comparable quality with traditional TV. As most mobile TV networks still are not much less than prototypes there is still some way to go before the transmission quality is sufficiently good.

This paper proceeds as follows: Section 2 introduces the theoretical foundation of this study. Section 3 outlines the key terms and technologies. Section 4 focuses on architectural model; Finally Section 5 summarizes the results of study.

\section{RELATED WORKS}

Media Cloud is a general idea of integrating the multimedia services with the cloud technologies, which enables the user to access the multimedia content through the cloud computing technology. Providing multimedia service through the cloud technology faces the scalability, Qos and heterogeneity challenges [4]. However the Content Delivery Network (CDN) and the peer to peer multimedia computing have been worked out to alleviate the problems in multimedia computing by pushing the multimedia content to the edges and to the peers respectively [4]. Rings et al has proposed the idea of integrating the cloud computing with the multimedia services without the Qos provisioning. Whereas Zhu et al proposed the multimedia cloud computing which provides Qos provisioning. The working of the cloud services for the multimedia content can be differentiated and explained mainly by two ways, they are: multimedia-aware cloud and cloud aware multimedia. Here the former idea pertains to the quality of service(QOS) services for multimedia content and the later concept deals about how well the sharing, retrieval and storage of the multimedia content can utilize the cloud-computing resources for achieving a better Quality Of experience [2] More finer framework like as Cloud Assisted Live Media Streaming have been studied to utilize the cloud resources by leasing and adjusting the cloud servers for the dynamic user demands[15].Basically the video streaming using the cloud technology relies on the transcoding mechanism, which has two mapping options like Hallsh-based and Lateness-first 
Mapping for reducing the jitter in the transcoding [7]. Also the Scalable Video Coding framework has also been proposed to reduce the latencies in the transcoding and to adapt to the dynamically changing network conditions [3]. The works of Satyanarayanan et al [13] have suggested the implementation of the dynamic virtual machine in carrying out the offloading to the mobile devices computations. The work of the proxy servers in the cloud technology for the multimedia or video streaming service has effectively handled the issue of the storage, processing challenges faced by some mobile devices in accessing those services [1] have seen the idea of interactive live streaming through the cloud computing technology, which provides a co-viewing experience to the users with their friends in different geographic locations. Thereby making the activity more social able. The experiments by Oehllberg et al [6] on the social activities of a human while viewing different video contents have been inspiring but still those frameworks cannot be applied in to the mobile environments directly. The design proposed by the Coppens et al [4] have been intended to elaborate the social interactions but it got constrained with the broadcast program channels. Reflex [7] is a mechanism to enhance spontaneity in the interaction between the users, which makes use of the Google App Engine to achieve more scalable and quality service in the social network, taking the large amount users into consideration. These interactions can be handled by the messenger service through the cloud computing technology.

The video streaming through the help of cloud computing technology has been facing another significant challenge as studied by [2], which is the Quality of experience [2] have already proposed the idea of enhancing the quality of experience in viewing the live video content by introducing the features like zooming, segmentation and panning but have not extended to the interactive streaming environment. Here we are proposing a framework for enriching the quality of experience to the users in an interactive live streaming, with the cloud computing as its backbone.

\section{CLOUD-BASED INTERACTIVE STREAMING}

The live video streaming has took a whole new dimension with the evolution of cloud computing technology. In the case of live video streaming, the cloud computing extends its support for interaction within the users and for a better quality of experience. The architecture workflow has been given as below in figure. The proxy servers or surrogates are assigned to each user who gets logged in to the cloud. These servers are provided by the Infrastructure as a service cloud. The surrogates will efficiently provide the offloading, it will be enacting as a middleware between the mobile devices and the video sources. It will be encapsulating the transcoding, segmentation and content adaptation operations. In addition to these operations the messenger, will also be handled by these surrogates, which is a key component in delivering effective interaction between the users.
The quality of experience for the users can be enhanced by the features like segmentation, zooming and content adaptations. The extensible messaging and presence protocol (XMPP), here plays a significant role in operating the transportation of the video segments and it is helpful in exchanging the metadata of these video streams like title, description.

\subsection{Transcoding}

The surrogates assigned to the users, will be handling the trancoder, which decide the encoding format for the video stream dynamically. The bit rate and the dimension of the video stream will also be decided in this module. Since MPEG4 has been the de-facto standard for the video delivery over loosy medium, this stream will be generally followed for the implementation. FFmpeg library is fundamentally utilized by this service for generating the thumbnails.

The transcoding frameworks splits the video contents into overlapping and non-overlapping group of pictures (GOP). These pictures may encircle different encoding qualities and resolutions, forming several layers.Such layers will be sliced as a coding-independent contents. Here 16x16 macro-blocks are framed for luma components and $8 \times 8 \mathrm{MB}$ is framed for chroma components. The granularity is decided with the parallelism in GOP level and MBs,which is divided into interparallelism and intra-parallelism.The simple diagramatic illustration in the fig [ ] of the transcoding framework is given below will give a better understanding of the idea elloborated above.

(i) Inter-node Parallelism: The Inter-node parallelism manages the reduced picture nodes. The real-time transcoding is achieved through this inter-node parallelism,the transcoding jitter is an effect of the variatons in the time delay of the encoding of the GOP(Group Of Pictures). The encoding time is mainly estimated for the optimization problem .

(ii) Intra-node Parallelism: The Intra-node parallelism manages the individual slice of the picture node. The Intranode parallelism is very significant in the GOP encoding and it is vital in fixing the upper bound on the average computation time spent on the GOP encoding. Moreover this parallelism is not simply enough in reducing the access time

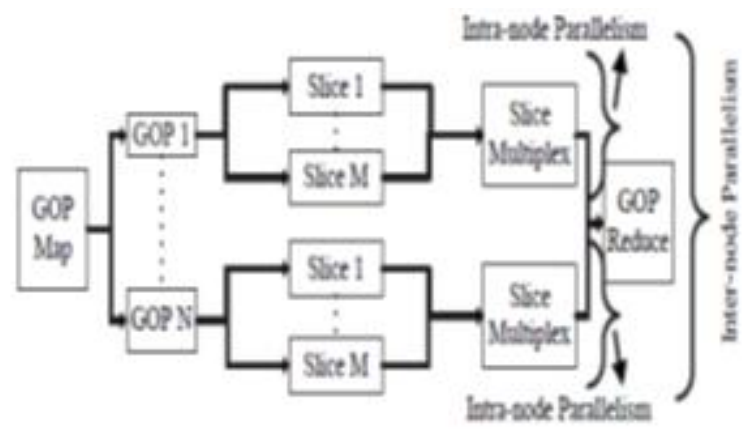

Fig. 1 Transcoding Framework 


\subsection{Segmentation}

The segmentation, is a feature to enhance the streamed videos, here this feature will create the list of points of scenes by identifying the scenes in the video. And by making use of the transcoding service the thumbnails for such segments will be created. Basically this feature is based on the OpenCV algorithms.

\subsection{Zooming}

This service will enable the users to zoom into the middle of the video stream. This will also be responsible in cropping of the streamed videos. This service fundamentally follows the object recognition service, which necessary in recognizing the objects in the video. The object recognizing service can enable to perform more complex zooming functionalities.

\subsection{Messenger}

This service is responsible in delivering the asynchronous message to the user from the surrogates. User will make queries periodically to the social cloud through this service, which is in connection with the social cloud. This service is also responsible in processing the plain text data, which are in $\mathrm{xml}$ format.

\subsection{Gateway}

The authentication of the users logged in are checked by this service, which also handles the logged in user list and maintains it in a separate database. The gateway is also responsible in reserving and destroying the surrogates based on the current work load.

\subsection{Social Cloud Service}

The social cloud service will be maintaining the data stores like Big-Table in Google App Engine, for storing all the details regarding the user records, sessions and messages. A data store interface is used to query and manipulate the Big-table which indeed a multi-dimensional maps, stores data object as an entity entitled with multiple properties. For the video streaming it fundamentally follows the RTP protocol. The XMPP handles the metadata of the video segments. The social cloud is basically extended by the PaaS services.

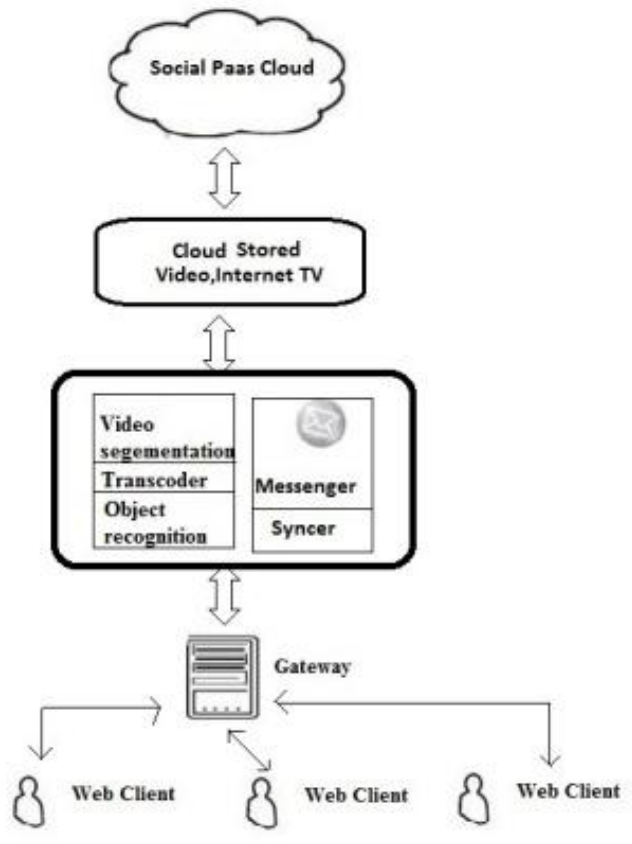

Fig.2 Architectural Framework

\subsection{Synchronizer}

The synchronizer features the property of enabling the user to view the concerned video content in the same window as the other user in same session. This is achieved by retrieving current playback position and guides the user to adjust to that position. This is particularly concerned in providing the coviewing experience to the users.

\section{PERFORMANCE METRICS}

In this proposed framework there are certain factors which affect its performance, where are here discussing some of the possible factors and the predicted performance improvements by this framework

\subsection{Transcoding Latency}

The Transcoding latency can make an impact on the entire framework if had not handled carefully. Even with the modern day's multi core processors the process of encoding is highly complex in nature. The transcoding delay can cause the delay in the access of video content for the user and it could cause even the freezing of the particular video content.

\subsection{Power Consumption}

The power consumption of the device, used for the access is significantly important, since that might well have a prime role in the working of this proposed framework in a considerable margin. The playback segment size of 10 , which has been widely followed in streaming applications, was proposed by 
the Http Live Streaming protocol. However this segment has found to have drained the battery life significantly. Normally the power consumption factor is profiled by an Xcode tool called "Instruments".

\subsection{Interaction Spontaneity}

In this proposed framework the spontaneity of the interaction between the connected users is measured by two levels of factors. The first is the latency in the sending of the message to the surrogates and the confirmation for the message being registered in the cloud. The other is the latency in query sent by the user to reach the assigned surrogates. Also the roundtrip time between the surrogates and the GAE which also need to be taken into consideration for calculating the interaction latency in this framework.

\section{IMPROVEMENTS PREDICTED WITH THE FRAMEWORK}

\subsection{Battery Efficiency}

To eliminate battery usage of the mobile devices this proposed architecture is aimed at providing an efficient burst transmission technique, which enables the mobile devices to operate on three states as High, Low and Intermediate. This technique fundamentally follows the Http Live streaming protocol, by which the video will be segmented by the surrogates and sent to the mobile devices on request. The mobile devices will be operating in High state when receiving the video segments and will be in Low state when remaining idle. The Intermediate state acts as the transition state between these two states.

\subsection{User Experience}

Another important key aspect of this framework is the enhancement of the user experience by the features like zooming in and out the streamed video and the Scene by scene segmentation of the video stream. This enhancement is particularly achieved with the help of the XMPP protocol and RTP protocol which handles the video streaming, exchange of the metadata and video segments information. Here the metadata handler will be responsible in fetching the video segments and Playlist handler will be responsible in providing the preview thumbnails of the videos. The thumbnails, having a smaller resolution will get loaded very fast. The object recognition service is a key part in providing the zooming feature on the streamed videos.

\subsection{Spontaneous Interactivity}

Interaction is a key aspect of a social live streaming framework and this aspect is effectively handled in this proposed architecture by the means of the Messenger service, which being operated in a asynchronous way will provide spontaneous interactions between the connected users. A Bigtable like datastore will be made use to handle these data.

\subsection{Scalability}

As this proposed framework takes the implementation phase, a challenge will be the ability of this entire system to handle the large amount users who gets too logged into the service (i.e.) the scalability measure of this framework. Being deployed on to the cloud network, it should effectively handle this problem as well but still this area has to be addressed in the future works.

\section{REMARKS AND FUTURE WORKS}

Our work has concerned primarily in suggesting a framework integrating the social interactive live streaming with the Quality enhanced user experience. The enhancement in the QoE of the user obviously shows great scope in the further feature enhancement with the streamed video contents, like as recording the live video contents to the device, noise removal and so on. On the other side the interactive aspect of the framework has several areas to be more deeply addressed like applying memcache support and more efficient transcoding mechanism.

\section{CONCLUSIONS}

We conclude by proposing a framework for enriching the quality of experience to the users in an interactive live streaming, with the cloud computing as its backbone. And mobile users can import a live or on-demand video to watch from any video streaming site, invite their friends to watch the video concurrently, and chat with their friends while enjoying the video.

\section{ACKNOWLEDGEMENTS}

The authors are immensely happy to extend their gratitude to the reviewers for their insightful thoughts, which this paper incorporates. We also thank the study participants for their Invaluable time and appreciable enthusiasm.

\section{REFERENCES}

[1] $\mathrm{Yu} \mathrm{Wu}$, Zhizhong Zhang, Chuan Wu, Zongpeng Li, Francis C.M. Lau:"Cloud MoV: Cloud-based Mobile Social TV", IEEE 2013.

[2] DejanKovachev, Yiwei Cao and Ralf Klamma,"Cloud Services for Improved User Experience in Sharing Mobile Videos", IEEE 2012.

[3] Zixia Huang, Chao Mei, Li Erran Li, Thomas Woo, "Cloud Stream delivering high-quality streaming videos through a cloud-based SVC proxy".

[4] Wen Huil, Chuang Lin and Yang, "Media -Cloud: A New Paradigm of Multimedia Computing", KSII: Transaction on Internet and Information Systems, Vol.6, April 2012. 
[5] Ramesh.B, Savitha.N, Manjunath.A.E,"Mobile Application in Multimedia Cloud Computing", Int.J.Computer Technology and Application, Vol 4(1), 97-103.Feb, 2013.

[6] Nicolas Ducheneaut, Robert.J.Moore1, Lora Oehlberg, James.D.Thornton, EricNickell,"SocialTV: Designing for Distributed, Sociable Television Viewing".

[7] Zimu Liu, Yuan Feng, Baochun Li,"Socialize Spontaneously with Mobile Applications",IEEE'12, INFOCOM proceedings.

[8] Hassnaa Moustafa and Nicolas Maréchal, Sherali Zeadally, "Mobile Multimedia Applications: Delivery Technologies", IEEE, CS (September/October 2012).

[9] H. Schwarz, D. Marpe, and T. Wiegand, "Overview of the scalable video coding extension of the H.264 AVC standard," IEEE Transaction on Circuits and Systems for Video Technology, vol. 17, no. 9, pp. 1103-1120, Sep. 2007.

[10] Z. Huang, C. Mei, L. E. Li, and T. Woo, "Cloud Stream: Delivering High-Quality Streaming Videos through a Cloud-Based SVC Proxy," in IEEE INFOCOM, 2011.

[11] Y. Wu, C. Wu, B. Li, X. Qiu, and F. C. Lau, "Cloud Media: When Cloud on Demand Meets Video on Demand," in IEEE ICDCS, 2011.

[12] Z.Wu, C.Zhang, Y. Ji, and H. Wang. Towards Cloud and Terminal Collaborative Mobile Social Network Service. 2010 IEEE International Conference

[13] M. Satyanarayanan, P. Bahl, R. Caceres, and N. Davies, "The case for vm-based cloudlets in mobile computing," IEEE Pervasive Computing, vol. 8, pp. 1423, 2009.

[14] A.Carroll and G.Heiser, "An analysis of power consumption in as Smartphone," in Proc. of USENIXATC, 2010.

[15] Feng Wang, Jiangchuan Liu, and Minghua Chen, CALMS: Cloud-Assisted Live Media Streaming Globalized Demands with Time/Region Diversities".

[16] R. Schatz and S. Egger, "Social Interaction Features for Mobile TV Services, "in Proc. of 2008 IEEE International Symposium on Broadband Multimedia Systems and Broadcasting, 2008.

[17] H. Knoche, M. Papaleo, M. A. Sasse, and A. VanelliCoralli, "The Kindest Cut: Enhancing the User Experience of Mobile TV Through Adequate Zooming," in Proceedings of the 15th International Conference on Multimedia, ser. MULTIMEDIA '07. ACM, 2007, pp. 87-96

[18] R. Pereira, M. Azambuja, K. Breitman, and M. Endler, "An Architecture for Distributed High Performance Video Processing in the Cloud," in Proceedings of the 2010 IEEE 3rd International Conference on Cloud Computing, 2010, pp. 482-489. 